\title{
Branched Polymer-Grafted Silica. Cationic Postgrafting from Pendant Acylium Perchlorate Groups of Grafted Polymer Chains on Ultrafine Silica Surface
}

\author{
Kazuhiro FujIKI, Nobuo MotoJi, Hideyo Tsuchida, ${ }^{*}$ \\ and Norio TsuBoKaWA* \\ Division of Life and Health Sciences, Joetsu University of \\ Education, 1, Yamayashiki, Joetsu, Niigata 943, Japan \\ *Department of Material and Chemical Engineering, \\ Faculty of Engineering, Niigata University, \\ 8050, Ikarashi 2-nocho, Niigata 950-21, Japan
}

(Received September 9, 1993)

\begin{abstract}
The cationic postgraft polymerization of several monomers initiated by pendant acylium perchlorate groups of polymer chains previously grafted onto a surface of ultrafine silica was investigated. The introduction of pendant acylium perchlorate groups to the grafted polymer chains on the silica surface was achieved by the reactions of silver perchlorate with pendant acyl chloride groups prepared by the radical graft copolymerization of styrene with acryloyl chloride using the ultrafine silica having azo groups onto the surface as an initiator. The pendant acylium perchlorate groups of the grafted copolymer chains on the silica surface were able to initiate the cationic postgraft polymerization of styrene, and polystyrene was effectively postgrafted from these grafted copolymer chains with a higher percentage of grafting to give branched polymer-grafted silica. The percentage of postgrafting of polystyrene and that of total polymer grafted onto silica, i.e., overall grafting, reached about 80 and $120 \%$, respectively. The cationic ring-opening polymerization of $\varepsilon$-caprolactone was also initiated by pendant acylium perchlorate groups of the grafted copolymer chains to give polyester-postgrafted silica. Postgrafting of polystyrene was remarkably affected by the composition of the copolymer previously grafted. The percentage of postgrafting increased with the solubility of these copolymer chains in the polymerization system. The polystyrene-postgrafted silica gave a stable colloidal dispersion in a good solvent for the grafted polymer.

KEY WORDS Ultrafine Silica / Surface Grafting / Branched Polymer /

Postgrafting / Cationic Polymerization / Acylium Perchlorate Group / Acyl

Chloride Group / Styrene / Lactone /
\end{abstract}

The dispersibility of inorganic ultrafine particles, such as silica, titanium oxide, and ferrite, into solvents or polymer matrices is remarkably improved by grafting of polymers onto these surfaces. ${ }^{1,2}$ In a series of papers, we reported the grafting of polymers onto these ultrafine particles initiated by surface azo, ${ }^{3}$ peroxide, ${ }^{4}$ potassium carboxylate, ${ }^{5}$ and acylium perchlorate ${ }^{6}$ groups. During these polymerizations, polymer-grafted ultrafine particles with a higher percentage of grafting were successfully obtained through the prop- agation of polymer chains from the particle surface. For instance, acylium perchlorate groups introduced onto a silica surface have ability to initiate the cationic polymerization of vinyl monomers and the cationic ringopening polymerization of lactones and cyclic ethers to give the corresponding polymergrafted ultrafine silica. ${ }^{6}$

On the other hand, the dispersibility and the wettability of these ultrafine particles are influenced by the percentage of grafting of polymers because the grafted polymer chains 
on the surface interfere with the aggregation of the particles and increase the affinity of the surface for solvents. ${ }^{2}$ Therefore, it is necessary to increase the percentage of grafting of polymers which have strong affinity for solvents in order to facilitate the dispersion of the ultrafine particles. The dispersion stability increases with rise in percentage of grafting of polymers, but becomes constant even at higher percentage of grafting. Accordingly, we examined the postgrafting to obtain branched polymer-grafted ultrafine particles in expectation of a more stable dispersion.

In previous papers, we reported the anionic postgrafting of polyester initiated by pendant potassium carboxylate groups of polymer chains ${ }^{7}$ and the radical postgrafting of vinyl polymers to unsaturated polyester chains ${ }^{8}$ previously grafted onto carbon black surface to give branched polymer-grafted carbon black with a higher percentage of grafting.

In this article, the introduction of pendant acylium perchlorate groups to copolymer chains previously grafted onto the silica surface (eq 1) and the cationic postgraft polymerization of several monomers initiated by these pendant acylium perchlorate groups (eq 2) are investigated. The effect of composition of the copolymer previously grafted onto silica

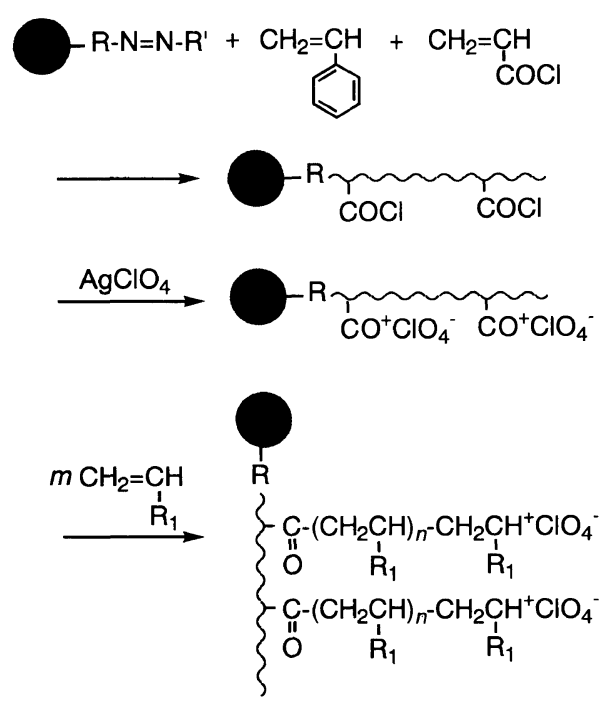


Grafting of Poly(styrene-co-AC) onto Silica Surface

The grafting of poly(styrene-co-AC) onto the silica surface was carried out by the copolymerization of styrene with AC initiated by the azo groups introduced onto the surface. A typical example is as follows. Freshly distilled styrene and AC were added into a flask containing $0.30 \mathrm{~g}$ of silica having azo groups and $20 \mathrm{~cm}^{3}$ of benzene. The reaction mixture was stirred at a constant rate at $60^{\circ} \mathrm{C}$ under dry nitrogen. After the reaction, the content of the flask was poured into a large excess of hexane to precipitate the copolymer containing the silica. The precipitate was extracted with benzene in order to remove ungrafted copolymer from the product. The isolated copolymergrafted silica was dried in vacuo at $45^{\circ} \mathrm{C}$. The pristine ungrafted silica particles could not be removed from the copolymer-grafted silica.

\section{Introduction of Pendant Acylium Perchlorate} Groups to Copolymer Chains Grafted on Silica Surface

Preparation of the silica having pendant acylium perchlorate groups in the grafted copolymer [poly(styrene-co-AC)] chains, i.e., pretreatment, was achieved by the reaction of silver perchlorate with the pendant acyl chloride groups of the grafted copolymer chains on the surface. The pretreatment was carried out as follows. Into a $100 \mathrm{~cm}^{3}$ flask, $0.20 \mathrm{~g}$ of poly(styrene-co-AC)-grafted silica, $0.20 \mathrm{~g}$ of silver perchlorate, and $5.0 \mathrm{~cm}^{3}$ of nitrobenzene were charged. The mixture was stirred with a magnetic stirrer under dry nitrogen at room temperature for $48 \mathrm{~h}$ because silver perchlorate is hardly soluble in nitrobenzene.

\section{Postgraft Polymerization}

After pretreatment, $20 \mathrm{~cm}^{3}$ of monomer was added to the above mixture containing the silica having pendant acylium perchlorate groups in the copolymer chains. Polymerization was conducted with stirring at $40^{\circ} \mathrm{C}$ under nitrogen, and after a given time, the contents of the flask were poured into excess methanol to precipitate the polymer containing the silica. The precipitate was collected by centrifuging, washed with methanol, and dried in vacuo at $45^{\circ} \mathrm{C}$.

The conversion was calculated by the following equation:

$$
\text { Conversion }(\%)=\frac{A}{B} \times 100
$$

$A$, Precipitate obtained (g)-Silica used (g)

$B$, Monomer used (g)

\section{Percentage of Postgrafting and Efficiency of} Postgrafting

To isolate the polymer-postgrafted silica from the reaction mixture containing ungrafted polymer, the product was dispersed in tetrahydrofuran and the dispersion was centrifuged at $1.0 \times 10^{4} \mathrm{rpm}$ until the silica precipitated completely. The silica precipitated was dispersed again in tetrahydrofuran and then centrifuged. This procedure was repeated until no polymer was detected in the supernatant solution. The isolated polymer-postgrafted silica was dried in vacuo at $45^{\circ} \mathrm{C}$. The percentage of postgrafting and the efficiency of postgrafting were calculated by the following equations:

$$
\begin{gathered}
\text { Postgrafting }(\%)=\frac{A}{B} \times 100 \\
\text { Efficiency }(\%)=\frac{A}{C} \times 100
\end{gathered}
$$

$A$, Polymer postgrafted (g)

$B$, Silica used (g)

$C$, Total polymer formed in the postgrafting (g)

The amount of polymer postgrafted was determined from the difference in weight of the silica before and after the postgraft polymerization.

\section{Dispersibility of Polymer-Grafted Silica}

Stability of the polymer-grafted silica dis- 
persion in organic solvents was estimated by the content of the silica particles in the dispersion. ${ }^{5}$ Polymer-grafted silica $(1.0 \mathrm{~g})$ was dispersed in $100 \mathrm{~cm}^{3}$ of tetrahydrofuran with a magnetic stirrer, and the dispersion was allowed to stand at room temperature. After a prescribed time, $5.0 \mathrm{~cm}^{3}$ of dispersion liquid was taken out with a pipet, dried in vacuo, and the residual silica particles were weighed. The percentage of silica dispersed was determined as follows:

$$
\text { Silica dispersed }(\%)=\frac{A}{B} \times 100
$$

$A$, Silica dispersed after standing (g)

$B$, Silica dispersed before standing $(\mathrm{g})$

\section{RESULTS AND DISCUSSION}

\section{Grafting of Poly(styrene-co-AC) onto Silica} Surface

We reported that azo groups introduced onto ultrafine inorganic particles or carbon black have ability to initiate the radical graft polymerization of vinyl monomers. ${ }^{3,11,12}$ In the polymerization, polymer-grafted ultrafine inorganic particles or carbon black is obtained through propagation of the polymer from the surface.

On the other hand, it has been reported that styrene and $\mathrm{AC}$ are copolymerized to give a reactive copolymer having pendant acyl chloride groups. ${ }^{13}$ Acyl chloride groups are known to be converted to acylium perchlorate groups by the reaction with silver perchlorate. Therefore, the copolymerization of styrene with AC initiated by azo groups introduced onto the silica surface was investigated to. obtain the copolymer-grafted silica having pendant reactive acyl chloride groups.

The results are shown in Table I. The copolymerization proceeded even in the absence of the silica having azo groups, but the rate of the copolymerization was considerably small. Untreated silica had no ability to initiate the copolymerization because the conversion in
Table I. Grafting of poly(styrene-co-AC) onto slica surface ${ }^{a}$

\begin{tabular}{|c|c|c|c|}
\hline In monomer & In copolymer & Time & Grafting \\
\hline Styrene/AC ${ }^{b}$ & Styrene/AC ${ }^{b}$ & $\mathrm{~h}$ & $\%$ \\
\hline $0.75 / 0.25$ & $0.75 / 0.25^{\mathrm{c}}$ & 2 & 23.1 \\
\hline $0.50 / 0.50$ & $0.57 / 0.43^{c}$ & 1.5 & 14.3 \\
\hline $0 / 1.00$ & $0 / 1.00$ & 30 & 20.5 \\
\hline
\end{tabular}

a Silica-R-N $=\mathrm{N}-\mathrm{R}^{\prime}, 0.20 \mathrm{~g}$, total monomer, $0.25 \mathrm{~mol}$; benzene, $30 \mathrm{~cm}^{3} ; 60^{\circ} \mathrm{C}$.

b Molar ratio.

c Determined by elemental analysis.

the presence of untreated silica was almost equal to that in the absence of the silica. The copolymerization of styrene with $\mathrm{AC}$ and the polymerization of $\mathrm{AC}$ alone were initiated by the azo groups introduced onto the surface, and the corresponding polymer was grafted onto the silica surface. The azo group content of the silica surface was determined to be $0.08 \mathrm{mmolg}^{-1}$ by elemental analysis. The polymerizations were all dead-end type. Conversion occurred in the range of $20-30 \%$, and did not increase even when the polymerization time was extended up to $4 \mathrm{~h}$.

The composition of the copolymer grafted onto the silica surface was determined by elemental analysis. It was nearly equal to the monomer-feed ratio though the rate of the copolymerization and the percentage of grafting were affected by a monomer-feed ratio. The monomer reactivity ratios of styrene and $\mathrm{AC}$ are reported to be 0.10 and 0.02 , respectively. ${ }^{13}$ The copolymer compositions of styrene/AC estimated from these monomer reactivity ratios are $0.56 / 0.44$ for $0.75 / 0.25$ in the feed, and $0.52 / 0.48$ for $0.50 / 0.50$ in the feed. The content of the AC moiety in the obtained poly(styrene-co-AC), however, was less than that calculated from the monomer reactivity ratios. The reason for this may be that the conversion reached more than $20 \%$ and that the copolymerizations were initiated by the radicals produced on the silica surface 
different from the conventional free radical initiation.

Based on the above results, it is concluded that the azo groups introduced onto the silica surface have ability to initiate the copolymerization of styrene with $\mathrm{AC}$ and that copolymers having pendant acyl chloride groups with various compositions can be prepared.

\section{Evidence of Initiation by Pendant Acylium Perchlorate Groups in Grafted Copolymer Chains}

It is well known that benzoyl or acetyl perchlorate prepared by reactions of acid chlorides with silver perchlorate has ability to initiate the cationic polymerization of styrene and the ring-opening polymerization of cyclic monomers. ${ }^{14}$ We reported the introduction of acylium perchlorate groups onto carbon black and silica surfaces. These acylium perchlorate groups were capable of initiating the cationic graft polymerization of vinyl monomers, ${ }^{6,15}$ and the cationic ring-opening polymerization of lactones, ${ }^{6,16}$ cyclic ethers, ${ }^{6,17,18}$ and cyclic acetals $^{19}$ to give the corresponding polymergrafted carbon black. Accordingly, it is expected that the pendant acyl chloride groups of poly(styrene-co-AC) chains grafted onto the silica surface react with silver perchlorate to give pendant acylium perchlorate groups which have initiating activity for the cationic postgraft polymerization.

The postgraft polymerization of styrene was carried out under several conditions with silver perchlorate and the poly(styrene-co-AC)grafted silica having pendant acyl chloride groups. The composition and the percentage of grafting of the poly(styrene-co-AC) used for subsequent experiments were $0.75 / 0.25$ of styrene/AC and $23.1 \%$, respectively. The results are summarized in TableII.

Styrene was not polymerized by silver perchlorate, untreated silica, or poly(styreneco-AC)-grafted silica alone (Nos. 1, 2, and 4). In addition, the polymerization could not be detected even when untreated silica was
Table II. Polymerization of styrene initiated under several conditions $\mathrm{s}^{\mathrm{a}}$

\begin{tabular}{|c|c|c|c|}
\hline No Silice & $\mathrm{AgClO}_{4}$ & $\begin{array}{l}\text { Conver- } \\
\text { sion }\end{array}$ & $\begin{array}{l}\text { Postgraft- } \\
\text { ing }\end{array}$ \\
\hline & g & $\%$ & $\%$ \\
\hline 1 None & 0.20 & 0 & - \\
\hline 2 Untreated & - & 0 & 0 \\
\hline 3 Untreated & 0.20 & 0 & 0 \\
\hline $\begin{array}{l}4 \text { Poly(styrene-co-AC)- } \\
\text { grafted }\end{array}$ & - & 0 & 0 \\
\hline $\begin{array}{l}5 \text { Poly(styrene-co-AC)- } \\
\text { grafted }\end{array}$ & 0.20 & 2.0 & 34.4 \\
\hline
\end{tabular}

a Pretreatment: silica, $0.20 \mathrm{~g} ; \mathrm{C}_{6} \mathrm{H}_{5} \mathrm{NO}_{2}, 5.0 \mathrm{~cm}^{3}$; room temp., $48 \mathrm{~h}$. Polymerization: styrene, $20 \mathrm{~cm}^{3} ; 40^{\circ} \mathrm{C} ; 8 \mathrm{~h}$.

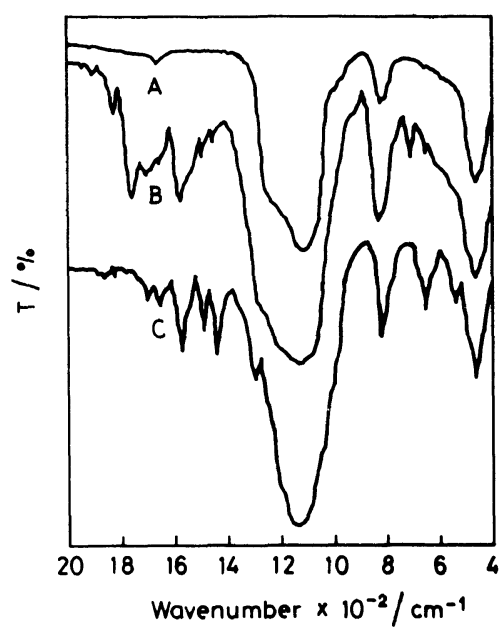

Figure 1. Infrared spectra of (A) untreated silica, (B) poly(styrene-co-AC)-grafted silica, and (C) polystyrenepostgrafted silica.

pretreated with silver perchlorate (No. 3).

The polymerization of styrene was initiated by the poly(styrene-co-AC)-grafted silica pretreated with silver perchlorate (No. 5) to give polystyrene-postgrafted silica. These results suggest that pendant acylium perchlorate groups are introduced onto the poly(styreneco-AC) chains previously grafted onto the silica surface by the treatment of the pendant acyl chloride groups of the copolymer with silver perchlorate and that postgrafted polystyrene chains propagate from the copolymer 
chains to give branched polymer-grafted silica.

Figure 1 shows the infrared spectra of untreated, poly(styrene-co-AC)-grafted, and polystyrene-postgrafted silica. The infrared spectrum of the polystyrene-postgrafted silica exhibited the disappearance of absorption at 1800 and $700 \mathrm{~cm}^{-1}$, which are characteristic of the acyl chloride group, and increased absorption at 1480 and $685 \mathrm{~cm}^{-1}$, which are characteristic of the phenyl group. These results clearly show the postgrafting of polystyrene from the poly(styrene-co-AC) chains previously grafted onto the silica surface.

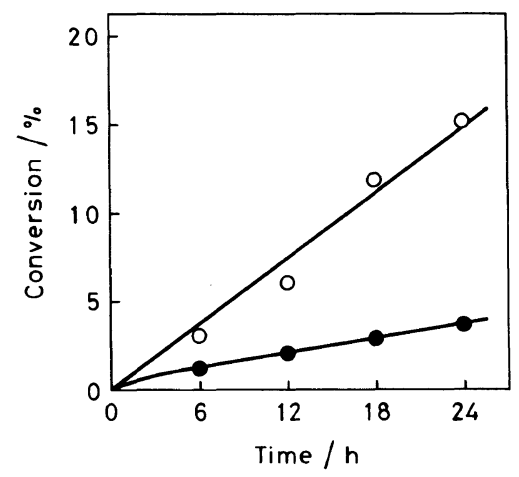

Figure 2. Cationic polymerization of styrene initiated by pendant acylium perchlorate groups of poly(styrene- $\mathrm{co}$ AC)-grafted silica. Pretreatment: poly(styrene-co-AC)grafted silica, $0.20 \mathrm{~g} ; \mathrm{C}_{6} \mathrm{H}_{5} \mathrm{NO}_{2}, 5.0 \mathrm{~cm}^{3} ; \mathrm{AgClO}_{4}, 0.20 \mathrm{~g}$; room temp., $48 \mathrm{~h}$. Polymerization: styrene, $20 \mathrm{~cm}^{3}$; (O), $40^{\circ} \mathrm{C}$; (O), $60^{\circ} \mathrm{C}$.

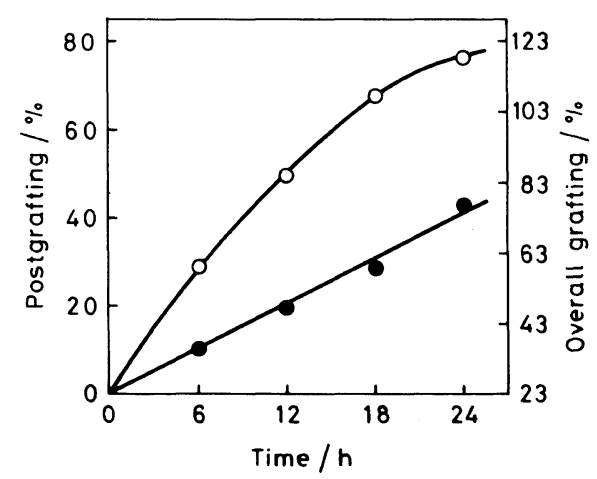

Figure 3. Relationship between polymerization time and percentage of postgrafting (overall grafting). Polymerization conditions are given in Figure 2. $(\odot), 40^{\circ} \mathrm{C} ;(\mathrm{O})$, $60^{\circ} \mathrm{C}$.

\section{Cationic Postgrafting}

Figures 2 and 3 show the time-conversion curves and the time-postgrafting (or overall grafting) curves for the polymerization of styrene, respectively. Overall grafting indicates the proportion of summational weight of the previously grafted poly(styrene-co-AC) and the postgrafted polystyrene to the weight of the silica used. Both conversion and percentage of postgrafting increased with polymerization temperature. The percentages of postgrafting and overall grafting at $60^{\circ} \mathrm{C}$ after $24 \mathrm{~h}$ reached 80 and $120 \%$, respectively. As previously reported, the percentages of grafting of polystyrene in the polymerizations initiated by azo, ${ }^{3}$ peroxide, ${ }^{4}$ and acylium perchlorate $^{6}$ groups on the silica surface reached $16.2,{ }^{3} 25.0,{ }^{4}$ and $64.5 \%,{ }^{6}$ respectively. Moreover, the percentage of postgrafting of polystyrene in radical postgrafting to unsaturated polyester chains previously grafted onto carbon black surface increased to about $40 \%{ }^{8}$ Consequently, the percentage of this cationic postgrafting value was very high.

Efficiencies of postgrafting at 40 and $60^{\circ} \mathrm{C}$ were $11-15$ and $6-11 \%$, respectively. Both efficiencies of postgrafting were high at the initial stage of the polymerization but decreased gradually as the polymerization proceeded. This suggests that the propagation of the postgrafted polymer occurs from the pendant acylium perchlorate groups and that an ungrafted polymer forms gradually by a chain transfer of the growing polymer cation to the monomer with progress of the polymerization. The efficiency of postgrafting at $60^{\circ} \mathrm{C}$ was somewhat lower than that at $40^{\circ} \mathrm{C}$. This is due to the fact that higher temperature tends to induce chain transfer of the growing polymer cation.

The results of the polymerization of $\mathrm{CL}$ are shown in Figure 4. The polymerization of CL was also found to be initiated by the pendant acylium perchlorate groups of the copolymer and give polyester-postgrafted silica with a higher percentage of grafting. The percentages 


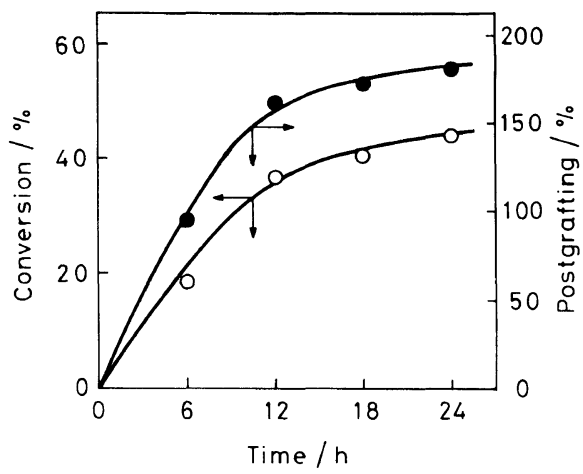

Figure 4. Cationic polymerization of $\mathrm{CL}$ initiated by pendant acylium perchlorate groups of poly(styrene-coAC)-grafted silica. Pretreatment: poly(styrene-co-AC)grafted silica, $0.20 \mathrm{~g} ; \mathrm{C}_{6} \mathrm{H}_{5} \mathrm{NO}_{2}, 5.0 \mathrm{~cm}^{3} ; \mathrm{AgClO}_{4}, 0.20 \mathrm{~g}$; room temp., $48 \mathrm{~h}$. Polymerization: $\mathrm{CL}, 20 \mathrm{~cm}^{3} ; 60^{\circ} \mathrm{C}$.

of postgrafting and overall grafting after $24 \mathrm{~h}$ reached 180 and $245 \%$, respectively.

\section{Effect of Composition of Copolymer Grafted onto Silica Surface}

As mentioned above, poly(styrene-co-AC) can be grafted onto the silica surface under various compositions, and its pendant acyl chloride groups can be converted to cationic initiating groups, acylium perchlorate groups, by the reaction with silver perchlorate. Therefore, we investigated how postgraft polymerization of styrene varies with the composition of the copolymer previously grafted onto the silica surface. The results are shown in Figures 5 and 6.

In the case of the poly(styrene-co-AC)grafted silica, both the rate of polymerization and the percentage of postgrafting increased with content of the AC moiety in the copolymer. When the poly(AC)-grafted silica was used, they decreased although the content of the AC moiety was more than that in the copolymer. This may be due to the fact that poly(AC) chains grafted onto the silica surface are sparingly soluble in the polymerization system because styrene is a poor solvent for poly(AC). Consequently, the pendant acylium perchlorate groups transformed from the pendant acyl chloride groups of the poly(AC)

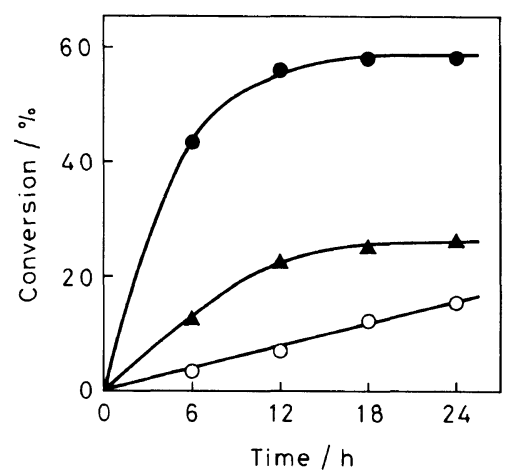

Figure 5. Effects of the composition of poly(styrene-coAC) grafted onto silica on the polymerization of styrene. Pretreatment: poly(styrene-co-AC)-grafted silica, $0.20 \mathrm{~g}$; $\mathrm{C}_{6} \mathrm{H}_{5} \mathrm{NO}_{2}, 5.0 \mathrm{~cm}^{3} ; \mathrm{AgClO}_{4}, 0.20 \mathrm{~g}$; room temp., $48 \mathrm{~h}$. Polymerization: styrene, $20 \mathrm{~cm}^{3} ; 60^{\circ} \mathrm{C}$. Composition of copolymer (styrene/AC): (O), 0.57/0.43; (O), 0.75/0.25; (A), 0/1.00.

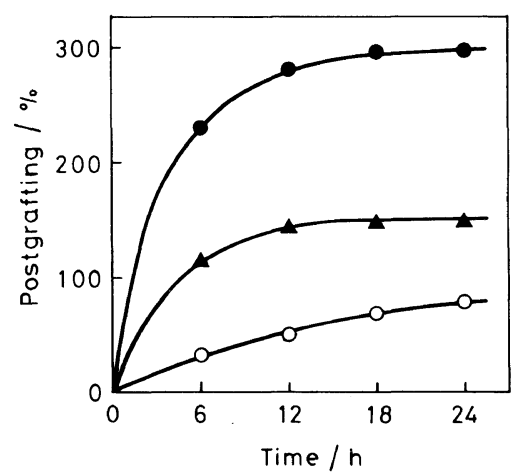

Figure 6. Relationship between polymerization time and percentage of postgrafting. Polymerization conditions are given in Figure 5. Styrene/AC: (O), 0.57/0.43; (O), $0.75 / 0.25 ;(\boldsymbol{\Delta}), 0 / 1.00$.

chains do not seem to be effectively used for the initiation of the polymerization. The postgraft polymerization of styrene using solvents to facilitate the dissolution of the poly(AC) chains in the polymerization system is now being examined.

\section{Stability of Polystyrene-Postgrafted Silica Dispersion \\ To confirm the usefulness of the postgraf- ting of polymers onto a silica surface, the stability of the polystyrene-postgrafted silica dispersion in tetrahydrofuran was compared}




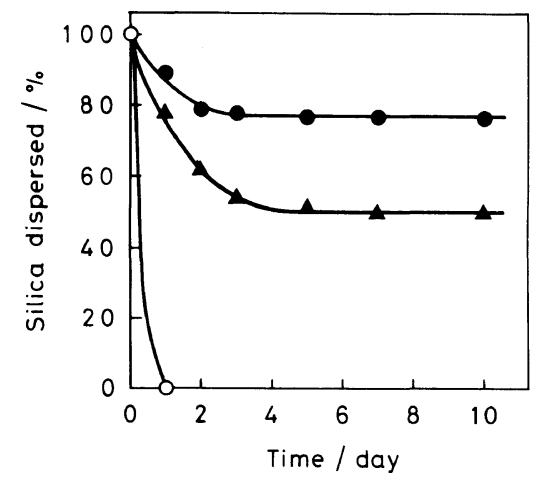

Figure 7. Stability of polystyrene-postgrafted silica dispersion in tetrahydrofuran at room temperature. (O), untreated silica; $(\boldsymbol{\Delta})$, poly(styrene-co-AC)-grafted silica (grafting $=23.1 \%) ;(\bigcirc)$, polystyrene-postgrafted silica (postgrafting $=80 \%$, overall grafting $=120 \%$ ).

with that of untreated and poly(styrene-coAC)-grafted ones. As demonstrated in Figure 7, untreated silica completely precipitated within 1 day. The precipitation of poly(styrene-co-AC)-grafted silica before postgrafting (grafting $=23.1 \%$ ) was somewhat delayed, but the polystyrene-postgrafted silica with a higher percentage of grafting (postgrafting= $80 \%$, overall grafting $=120 \%$ ) gave the most stable colloidal dispersion in tetrahydrofuran. This evidently indicates that the dispersibility of ultrafine silica in organic solvents is greatly improved by grafting of polymers onto the surface.

\section{CONCLUSIONS}

1. The transformation of the pendant acyl chloride groups of poly(styrene-co-AC) chains grafted onto the silica surface into the pendant acylium perchlorate groups was successfully achieved by the reaction with silver perchlorate.

2. The cationic polymerization of styrene and the ring-opening polymerization of $\mathrm{CL}$ were initiated by the pendant acylium perchlorate groups of the copolymer chains previously grafted onto the surface, and the corresponding polymer postgrafted with a higher percentage of grafting to give branched polymer-grafted silica.

3. The postgraft polymerization of styrene was considerably affected by the composition of the polymer chains previously grafted onto the silica surface.

4. The stability of polymer-postgrafted silica dispersion in good solvents for the grafted polymer chains was remarkably enhanced.

Acknowledgments. This work was partly supported by the Ministry of Education, Science, and Culture of Japan under Grant to Norio Tsubokawa.

\section{REFERENCES}

1. N. Tsubokawa and T. Endo, Tanso, No. 140, 322 (1989).

2. N. Tsubokawa, Prog. Polym. Sci., 17, 417 (1992).

3. N. Tsubokawa, A. Kogure, K. Maruyama, Y. Sone, and M. Shimomura, Polym. J., 22, 827 (1990).

4. N. Tsubokawa and H. Ishida, Polym. J., 24, 809 (1992).

5. N. Tsubokawa, A. Kogure, and Y. Sone, J. Polym. Sci., A, Polym. Chem., 28, 1923 (1990).

6. N. Tsubokawa and A. Kogure, Polym. J., 25, 83 (1993).

7. N. Tsubokawa, K. Fujiki, and T. Sasaki, Kobunshi Ronbunshu, 50, 235 (1993).

8. N. Tsubokawa, K. Fujiki, and Y. Sone, Kobunshi Ronbunshu, 44, 605 (1987).

9. M. Sato, Y. Kanbayashi, K. Kobayashi, and Y. Sima, J. Catalysis, 1, 342 (1976).

10. S. Matsuda and S. Okazaki, Nippon Kagaku Kaishi, 1287 (1986)

11. K. Fujiki, N. Tsubokawa, and Y. Sone, Polym. $J_{\text {., }}^{*}$ 22, 661 (1990).

12. K. Fujiki, N. Tsubokawa, and Y. Sone, J. Macromol. Sci.-Chem., A28, 715 (1991).

13. G. Smets and E. Dysseleer, Makromol. Chem., 91, 160 (1966).

14. P. Dreyfuss and J. P. Kennedy, J. Polym. Sci., Polym. Lett. Ed., 14, 139 (1976).

15. N. Tsubokawa, J. Polym. Sci., Polym. Chem. Ed., 22, 1515 (1984).

16. N. Tsubokawa, J. Appl. Polym. Sci., 30, 2041 (1985).

17. N. Tsubokawa, H. Nunokawa, and Y. Sone, $J$. Macromol. Sci.-Chem., A23, 105 (1986).

18. N. Tsubokawa, Yu Jian, and Y. Sone, J. Polym. Sci., A, Polym. Chem., 26, 2715 (1988).

19. N. Tsubokawa, J. Polym. Sci., A, Polym. Chem., 25, 1979 (1987). 